CERN-TH-2002-213

FAU-TP3-02-24

\title{
Wilsonian flows and background fields
}

\author{
Daniel F. Litim $\$$ and Jan M. Pawlowski \\ * Theory Division, CERN \\ CH-1211 Geneva 23. \\ ${ }^{\dagger}$ Inst. für Theoretische Physik III, Universität Erlangen, \\ Staudtstraße 7, D-91054 Erlangen, Germany.
}

\begin{abstract}
We study exact renormalisation group flows for background field dependent regularisations. It is shown that proper-time flows are approximations to exact background field flows for a specific class of regulators. We clarify the rôle of the implicit scale dependence introduced by the background field. Its impact on the flow is evaluated numerically for scalar theories at criticality for different approximations and regularisations. Implications for gauge theories are discussed.
\end{abstract}

\footnotetext{
*Daniel.Litim@cern.ch

†jmp@theorie3.physik.uni-erlangen.de
} 


\section{Introduction}

Renormalisation group ( $R G$ ) techniques are pivotal for the study of theories which are strongly coupled and/or have diverging correlation length. For practical purpose renormalisation group flows should be easily accessible by analytical means and should have good numerical stability within given truncations. Such flows provide a promising starting point for the study of more involved theories like (non-)Abelian gauge theories or even quantum gravity. The formulation of gauge theories is simplified by using background fields. Within the ERG approach background field techniques have been applied to non-Abelian gauge the-

ories [1,2, 3, 4, 5, 6, 6, 8, 8, 9, 10, 11, 12, 13, 14, to the superconducting phase transition [15, 16 and to a study of UV fixed points of Euclidean quantum gravity [17.

The full power of the background field method can be exploited by identifying the background field with the mean field. Then, background field flows offer remarkable simplifications for both analytical and numerical implementations. Analytic methods like heat kernel techniques are applicable and simplify the evaluation of the operator traces [1, 2, 11, 13, 14, However, identifying the background field with the mean field entails an inherent approximation on the flow: the outcome of an integration step provides an approximation to the input for the next integration step. Proper-time flows [18] are subject to a similar approximation [19,20,21], the main difference being that an additional contribution to the flow due to the field dependence of the regularisation is also neglected [21]. For these reasons, it is important to investigate the implications and limits of these approximations, both for ERG flows with background fields, and related generalised proper time flows.

In the present work we address the details of such an approach. We sketch the derivation of a general background field flow in scalar theories, which serve as a testing ground for the properties and limits of the background field approach. We detail their connection to proper time flows derived in [21] and discuss the inherent approximation from a formal point of view. These approximations are linked to terms proportional to the scale derivative of the full inverse propagator. Their impact is studied numerically by a calculation of critical exponents in $3 d$ scalar theories to leading order in a derivative expansion. Our results for different regularisations and approximations are discussed in the light of their conceptual differences. Implications for gauge theories are emphasised at various places.

\section{Generalities}

We briefly summarise the key steps of the derivation of the flow equation within the background field formulation. Consider the generating functional of a scalar theory with classical action $S[\phi]$ and a cut-off term $\Delta S_{k}[\phi, \bar{\phi}]=\frac{1}{2} \int(\phi-\bar{\phi}) R(\phi-\bar{\phi})$. Typically, the infrared regulator $R$ tends to a mass for small momenta $p^{2} / k^{2} \ll 1$ and decays exponentially for large momenta $p^{2} / k^{2} \gg 1$. In $\Delta S_{k}$, $\phi$ is the full field and the background field $\bar{\phi}$ is an auxiliary field at our disposal. A common choice for the background field $\bar{\phi}$ is the mean field: 
$\bar{\phi}=\langle\phi\rangle_{J}$. Note, that in gauge theories formulated in background field dependent gauges even the gauge fixed action $S$ depends on both, $\phi$ and $\bar{\phi}$. The full field $\phi=\bar{\phi}+(\phi-\bar{\phi})$ is split into the background field $\bar{\phi}$ and the fluctuation field $(\phi-\bar{\phi})$. In general, only these fields transform homogeneously under renormalisation group transformations. We allow for background field dependent regulator $R$ and define the regularised generating functional

$$
Z_{k}[J, \bar{\phi}]=\int d \phi \exp \left(-S[\phi]-\Delta S_{k}[\phi, \bar{\phi}]+\int J(\phi-\bar{\phi})\right)
$$

The propagating field is the fluctuation field $(\phi-\bar{\phi})$, which is coupled to the external current. The flow equation for the generating functional in (1) is given by $\partial_{t} Z[J, \bar{\phi}]=$ $-\left\langle\partial_{t} \Delta S_{k}[\phi, \bar{\phi}]\right\rangle_{J}$. This equation depends linear on the propagator of the fluctuation field $(\phi-\bar{\phi})$, as necessary for one loop exact flows [21]. The flow of $Z_{k}$ can be turned into a flow for the effective action $\Gamma_{k}[\phi, \bar{\phi}]=\int J(\phi-\bar{\phi})-\ln Z[J ; \bar{\phi}]+\Delta S_{k}[\phi, \bar{\phi}]$ :

$$
\partial_{t} \Gamma_{k}[\phi, \bar{\phi}]=\frac{1}{2} \operatorname{Tr} \frac{1}{\Gamma^{(2,0)}[\phi, \bar{\phi}]+R} \partial_{t} R,
$$

where

$$
\Gamma^{(n, m)}[\phi, \bar{\phi}]=\frac{\delta^{n}}{(\delta \phi)^{n}} \frac{\delta^{m}}{(\delta \bar{\phi})^{m}} \Gamma_{k}[\phi, \bar{\phi}] .
$$

The flow (2) is both consistent and complete, in the sense coined in [21]. It is a straightforward task to show that standard perturbation theory is contained in the integrated flow (2). For these considerations, the background field acts as a spectator. The next question is how the effective action depends on $\phi$ and $\bar{\phi}$. If $R$ is independent of $\bar{\phi}$, the effective action only depends on the full field $\phi$. In case we take a $\bar{\phi}$-dependent $R$, the effective action is not a functional of the full field $\phi$, but a functional of both fields. This can be summarised in the equation

$$
\Gamma_{k}^{(0,1)}[\phi, \bar{\phi}]=\frac{1}{2} \operatorname{Tr} \frac{1}{\Gamma^{(2,0)}[\phi, \bar{\phi}]+R} \frac{\delta R}{\delta \bar{\phi}},
$$

which controls the background field dependence of the effective action. Eq. (3) resembles the flow equation (2). The term on the right-hand side vanishes for $R \rightarrow 0$. In gauge theories the situation is slightly more complicated. As mentioned before, the action $S$ depends on both, the fluctuation field and the background field, in background field dependent gauges. Consequently (3) receives additional contributions from $\left\langle S^{(0,1)}(\phi, \bar{\phi})\right\rangle \neq 0$. These terms are even present for vanishing regulator $R=0$, e.g. [6,8,10]. An exception is provided by axial gauges, where the background field only enters the regulator term [14].

Now we want to find a particularly simple form of the flow (2), subject to an appropriate choice of the class of regulators $R$. The flow (2) depends on the two operators $R$ and $\Gamma_{k}^{(2,0)}[\phi, \bar{\phi}]$. For all purposes, in particular for its numerical treatment, we would like to choose a regulator $R$ which commutes with $\Gamma^{(2,0)}[\phi, \bar{\phi}]$, or at least comes as close as it 
gets. The obvious choice would be to take $R$ as a function of $\Gamma_{k}^{(2,0)}[\phi, \bar{\phi}]$. However, the dependence on $\phi$ implies that (2) would then contain additional flow terms to any loop order, as $\Gamma_{k}^{(2,0)}[\phi, \bar{\phi}]$ depends on arbitrary powers of $\phi$. This spoils the applicability of the flow. To maintain (2), the regulator $R$ only can depend on $\Gamma_{k}^{(2)}[\bar{\phi}, \bar{\phi}]$. There are other choices, guided by renormalisation group arguments, which lead to regulators that depend only linearly on $\Gamma_{k}^{(2,0)}[\bar{\phi}, \bar{\phi}]$, see [11]. We parametrise the regulators as

$$
R(\bar{x})=\bar{x} r(\bar{x})
$$

and define

$$
x:=\Gamma_{k}^{(2,0)}[\phi, \phi]
$$

and $\bar{x}=x[\phi=\bar{\phi}]$. The corresponding flow is

$$
\partial_{t} \Gamma_{k}[\phi, \bar{\phi}]=\frac{1}{2} \operatorname{Tr} \frac{1}{\Gamma^{(2,0)}[\phi, \bar{\phi}]+R[\bar{x}]}\left(\partial_{\bar{x}} R[\bar{x}] \partial_{t} \bar{x}-2 \bar{x}^{2} \partial_{\bar{x}} r[\bar{x}]\right)
$$

Eq. (6) can be used in two ways. First we can expand it about a background field $\bar{\phi}$ which has physical importance, e.g. the vacuum configuration. Such a procedure should stabilise the flow as already the chosen expansion points contains some non-trivial physical information. Of course this means that this information has to be at hand. Second, we can use (6) at $\bar{\phi}=\phi$, where the background field is the mean field. In this case we deal with the flow of the effective action

$$
\Gamma_{k}[\phi]:=\Gamma_{k}[\phi, \phi]
$$

Then, the flow on the right-hand side of (6) solely depends on the propagator $\Gamma_{k}^{(2,0)}[\phi, \phi]$ and its $t$-derivative. Inserting this in (6) at $\bar{\phi}=\phi$ we arrive at

$$
\partial_{t} \Gamma_{k}[\phi]=-\operatorname{Tr} \frac{x r^{\prime}}{1+r}+\frac{1}{2} \operatorname{Tr} \frac{r+x r^{\prime}}{x(1+r)} \dot{x}
$$

where $\dot{x}=\partial_{t} x$ and $r^{\prime}=\partial_{x} r$. We rush to add that (\$) is not closed. The propagator $\Gamma^{(2,0)}[\phi, \phi]$ is that of the fluctuation field, which is not identical to

$$
\Gamma_{k}^{(2)}[\phi]=\Gamma^{(2,0)}[\phi, \phi]+2 \Gamma^{(1,1)}[\phi, \phi]+\Gamma^{(0,2)}[\phi, \phi]
$$

for $R \neq 0$. This can be seen from (3). The same holds true for background field flows of gauge theories in axial gauges [14]. For gauge theories within background field dependent gauges, the situation is even more involved, since the cut-off term is not the only source for a non-vanishing right-hand side of (3). From now on we discuss the consequences of

$$
\Gamma_{k}^{(2)}[\phi] \stackrel{!}{=} \Gamma_{k}^{(2,0)}[\phi, \phi]
$$


which is equivalent to the requirement

$$
2 \Gamma_{k}^{(1,1)}[\phi, \phi]+\Gamma_{k}^{(0,2)}[\phi, \phi] \stackrel{!}{=} 0 .
$$

It has to be seen, what results can be obtained within such an approximation and where it approaches its limits. We emphasise that (9) is trivially satisfied without background fields. Furthermore, in scalar theories and gauge theories within axial gauges [14, the background field only enters the regulator term. Hence, (3) vanishes in the infra-red limit which guarantees that (9) is automatically satisfied in the same limit. Applications of the flow (8) to gauge theories or gravity in the approximation (9) and neglecting flow terms $\sim \dot{x}$ have been studied in [1,2, 15, 16, 17, , while the inclusion of flow terms $\sim \dot{x}$ has been considered in [13].

\section{Exact proper time flows}

Next, we discuss the link of (8) to the proper time renormalisation group (PTRG). It was shown in 20,21], that PTRG flows are not exact. For the derivation we follow the arguments detailed there: a general PTRG flow at an effective cut-off scale $k^{2}$ can be expanded in a set of flows

$$
\partial_{t} \Gamma_{k}[\phi]=\operatorname{Tr}\left(\frac{k^{2}}{k^{2}+x / m}\right)^{m}
$$

Here, $x=\Gamma_{k}^{(2)}[\phi]$ in the spirit of (5). The parameter $m$ describes different implementations of the proper-time regularisation. A general PTRG flow can be seen as a linear combinations of flows with different $m$. Another difference to (5) stems from the fact, that the proper time flow is derived without relying on background fields. In 21] we showed, that the proper time flows (10) represent approximations to exact background field flows and devised an exact generalised proper time flow. Eq. (10) lacks additional terms proportional to $t$ derivatives of $\Gamma_{k}^{(2)}$. Moreover it relies on the approximation (9). Now we present the corresponding $R$ with the leading term as in (10). We choose a regulator $R$ with $r[x]$ given by

$$
r_{m}[x]=\exp \left(\frac{1}{m}\left(\frac{m k^{2}}{x}\right)^{m}{ }_{2} F_{1}\left[m, m ; m+1 ;-\frac{m k^{2}}{x}\right]\right)-1 .
$$

The function $r_{m}$ is a solution of the differential equation

$$
\frac{x r^{\prime}}{1+r}=-\left(1+\frac{x}{m k^{2}}\right)^{-m}
$$

This equation comes from matching the ERG flow -by neglecting the terms proportional to $\partial_{t} \Gamma_{k}^{(2)}$ - to the proper time flow (10). The flow equation (8) with the regulator function $r$ as in (11) takes the form

$$
\partial_{t} \Gamma_{k}=\operatorname{Tr}\left(\frac{k^{2}}{k^{2}+x / m}\right)^{m}+\frac{1}{2} \operatorname{Tr}\left[\frac{r_{m}}{x\left(1+r_{m}\right)}-\left(\frac{k^{2}}{k^{2}+x / m}\right)^{m} \frac{1}{x}\right] \dot{x} .
$$


Eq. (12) reduces to (10) if the term proportional to $\dot{x}$ on the right hand side is dropped. An interesting case is provided by the limit $m \rightarrow \infty$, where $\left(1+x /\left(m k^{2}\right)\right)^{-m} \rightarrow \exp \left(-x / k^{2}\right)$. In this limit $r[x]$ takes the form

$$
r_{\infty}[x]=\exp \left(-\operatorname{Ei}\left[-\frac{x}{k^{2}}\right]\right)-1 .
$$

The flow for the regulator $r_{\infty}$ reads

$$
\partial_{t} \Gamma_{k}=\operatorname{Tr} \exp \left(-\frac{x}{k^{2}}\right)+\frac{1}{2} \operatorname{Tr}\left[1-\exp \left(-\frac{x}{k^{2}}\right)-\exp \left(\operatorname{Ei}\left[-\frac{x}{k^{2}}\right]\right)\right] \frac{1}{x} \dot{x} .
$$

Eq. (14) boils down to the $m \rightarrow \infty$ limit of the proper time flow (10), when neglecting $\dot{x}=\partial_{t} \Gamma_{k}^{(2)}$.

To conclude, apart from terms proportional to $\dot{x}=\partial_{t} \Gamma_{k}^{(2)}$ the ERG flows (12) resemble the proper time flows (10). Therefore, a general proper time flow like (10) is the approximation to a background field ERG flow, where $(i)$ terms proportional to $\dot{x}$ are neglected and $(i i)$ implicitly the difference between fluctuation field and background field has been neglected, resorting to (9).

This suggests to compare results of the (non-exact) PTRG to results from flows with the regulator argument $\Gamma_{k}^{(2)}$. Here, one can use optimisation criteria for this set of regulators [22]. Note that the choice (11) was only one particular example of the general set of $\Gamma_{k}^{(2)}{ }_{-}$ dependent regulators. Applications of the proper-time RG are based on flows (10), and approximations thereof. It would be interesting to see how these results are affected by the additional flow terms as given in (12).

The above analysis is also linked to a further observation made in [21. The flows (10) and (12) can be matched to generalised Callan-Symanzik flows. The difference has two sources: First, the flows deviate by terms proportional to $\partial_{t} \Gamma_{k}^{(2)}$ and higher scale derivatives of $\Gamma_{k}$ itself. Second, the Callan-Symanzik flow is defined without background fields and the argument $x=\Gamma_{k}^{(2)}$ is just the propagator of the full field. This has to be compared to (12), which is not a closed equation, and to (10), which is not exact. This leaves us with a representation of the missing terms made by the approximation (9) without resorting to background fields at all.

\section{Implicit scale dependences}

Up to now, we have discussed the implications of using a background field dependent regularisation. In general, we are led to flows that contain terms proportional to $\partial_{t} \Gamma^{(2)}$. A priori, it is difficult to estimate the numerical importance of those additional flow terms. Moreover, the validity of the approximation (9) cannot be taken for granted. Hence, it would be most useful to gain some intuition for these matters, in particular in view of future numerical applications. Here, we study the relevance of $\partial_{t} \Gamma^{(2)}$ terms for $O(N)$ symmetric scalar theories at criticality in three dimensions, using ERG flows with background fields in different approximations. In the following we discuss flows for regulators of the form 


$$
R=\left(k^{2}(1-X)-q^{2}\right) \theta\left(k^{2}(1-X)-q^{2}\right),
$$

where $q^{2}$ is plain momentum squared and $X$ encodes some intrinsic scale or background field dependence. For $X=0$, it reduces to an optimised regulator as introduced in [22]. We consider the flow equation for the effective potential $U_{k}$ to leading order in the derivative expansion. The flow equation is best written in dimensionless variables $u=U_{k} k^{-d}$ and $\rho=\frac{1}{2} \phi^{a} \phi_{a} k^{2-d}$. For the choice (15) of the regulator, the momentum integration can be performed analytically and leads to

$$
\begin{aligned}
\partial_{t} u+d u-(d-2) \rho u^{\prime}= & (N-1) \frac{\left(1-X_{1}\right)^{d / 2}\left(1-X_{1}-\frac{1}{2} \partial_{t} X_{1}\right)}{1-X_{1}+u^{\prime}} \theta\left(1-X_{1}\right) \\
& +\frac{\left(1-X_{2}\right)^{d / 2}\left(1-X_{2}-\frac{1}{2} \partial_{t} X_{2}\right)}{1-X_{2}+u^{\prime}+2 \rho u^{\prime \prime}} \theta\left(1-X_{2}\right)
\end{aligned}
$$

where $X_{1}, X_{2}$ are Goldstone and radial parts of the matrix $X$ in field space. For simplicity, we have rescaled the irrelevant factor $4 v_{d} / d$ on the right-hand side of (16) into the potential and the fields.

Since $X$ is at our disposal it can be used to enhance the stability of the flow. The standard case $X=0$ (plain momentum regularisation) has been discussed in [23]. We discuss the following natural choices for $X$ :

$A$ One can consider a regularisation which naturally includes an expansion about the minimum of the effective potential. We choose $q^{2}+X=\Gamma_{k}^{(2,0)}\left[\phi_{0}, \phi_{0}\right]\left(q^{2}\right)$. Additionally, we take $\phi_{0}=0$, which defines the $k$-dependent mass $m_{k}^{2}$ at vanishing field. We have

$$
X_{1}=X_{2}=m_{k}^{2} / k^{2} .
$$

The flow (16) with the choice (17) is denoted as flow $A$. Here, the regularisation introduces additional scale-dependent contributions to the flow, triggered by the running of the mass term at vanishing field. In consequence, no explicit background field dependence is introduced due to the regulator.

$B$ A second choice for $X$ is given by $q^{2}+X=\Gamma_{k}^{(2,0)}[\phi, \phi]$, the full field-dependent propagator. This entails that our flow is essentially diagonal, the operator trace only involves the full propagator and its scale derivative. However, this forces us to concentrate on the flow of $\Gamma_{k}[\phi]$ as defined in the previous section in (7) within the approximation (9). We have

$$
X_{1}=u^{\prime}(\rho), \quad X_{2}=u^{\prime}(\rho)+2 \rho u^{\prime \prime}(\rho) .
$$

The flow (16) with the choice (18) is denoted as flow $B$. Notice that the choice (18) corresponds to a cutoff in momenta and a cutoff in field amplitude, because the flow is suppressed once $\Gamma_{k}^{(2)}>k^{2}$. In contrast to flow $A$, the regularisation of the flow $B$ depends explicitly on the background field. 
$C$ Finally, we also compare the flows $A$ and $B$ to the exact proper time flow (14) for $m=\infty$. The operator $X$ is given by (18), and the flow is referred to as the flow $C$. It differs from flow $B$ only in the choice for the regularisation.

The structure of the flow (16) depends significantly on the choices $X=0$, (17) or (18). For $X=0$, the flow has a potential pole when $u^{\prime}$ or $u^{\prime}+2 \rho u^{\prime \prime}$ approach -1 . This is a direct consequence of the IR regularisation. For (17), the pole is shifted away because the choice for $X$ corresponds precisely to the most negative value for $u^{\prime}$ or $u^{\prime}+2 \rho u^{\prime \prime}$, respectively. This is even more pronounced for the field-dependent choice (18), because the denominator in (16) become trivial and field-independent. Still, the additional flow terms on the right-hand side of (16) imply potential poles, e.g. [23].

Next, we study the impact of the implicit scale dependence on the flow. This is done by partially considering the additional terms coming from the scale derivatives of $X$ in (16). The full scale derivative is proportional to

$$
k^{-2} \partial_{t}\left(k^{2} X\right)=2 X+\partial_{t} X
$$

The first term on the right-hand side displays the explicit scale dependence of $\sim k^{2}$, whereas the second term contains the implicit scale dependence of $X$ bound to vanish at a fixed point. Our first approximation is based on dropping the entire scale derivative of $k^{2} X$ by setting

$$
k^{-2} \partial_{t}\left(k^{2} X\right)=0 \text {. }
$$

This is referred to as flows $A^{\prime}, B^{\prime}$ and $C^{\prime}$, the prime indicating the additional approximation (20) on $A, B$ and $C$. In particular, the flow $C^{\prime}$ reads

$$
\partial_{t} u+d u-(d-2) \rho u^{\prime}=(N-1) \exp \left(-u^{\prime}\right)+\exp \left(-u^{\prime}-2 \rho u^{\prime \prime}\right)
$$

where we have absorbed an irrelevant numerical factor $2 v_{d}$ into the fields. This flow has been studied numerically in 24, 19. intrinsic dependence on $\partial_{t} X$, defined by

$$
k^{-2} \partial_{t}\left(k^{2} X\right)=2 X
$$

This is referred to as flows $A^{\prime \prime}, B^{\prime \prime}$ and $C^{\prime \prime}$, the double-prime indicating the additional approximation (22).

On a fixed point, we have $\partial_{t} X=0$, because $X$ is a dimensionless function. Using (19) and (22), we conclude that the fixed point solutions of the unprimed and the double-primed flows are identical. More generally, the fixed point solutions of any two flows are identical,

\footnotetext{
${ }^{\ddagger} 3$ d critical exponents have also been computed from (10) for other values of $m$, e.g. 25, 26, 19.
} 
if the flows differ only by a dimensionless flow term, which vanishes on a fixed point. For the $A$-flows, the function $X$ is field-independent. From the explicit form of the flows $A$ and $A^{\prime}$, we conclude that the non-universal fixed point solutions $\partial_{t} u=0$ are related by a simple rescaling of the fields and the effective potential. An analogous statement cannot be made about universal critical exponents. Indeed, critical exponents describe the approach towards the critical point. In the vicinity of the fixed point, the RG trajectories are strongly sensitive to the functions (19), (20) or (22). Despite the close similarity or even equality of the fixed point solutions, in general their critical exponents are all different.

\section{Results}

In Tab. 1, we have computed the critical exponent $\nu$ for the flows $A, A^{\prime}, A^{\prime \prime}, B^{\prime}, B^{\prime \prime}$ and $C^{\prime}$ for all $N$ between -2 and $\infty$. Results have been given for the physically most interesting cases $N=0,1,2,3$ and 4 . The critical exponent $\nu$ describes the approach towards the critical point. In the vicinity of the fixed point, the RG trajectories are strongly sensitive to the explicit form of the flow, which is quite different for the different cases introduced above. For $N=-2$, the critical exponent is known to be $\nu=\frac{1}{2}$. The universal large- $N$ result reads $\nu=1$. All flows in all approximations reproduce the known result at $N=-2$. Except for the flows $A^{\prime}$ and $A^{\prime \prime}$, the same holds also true for the large- $N$ limit. All results given in Tab. 1 are within $20 \%$ of the physical values, and some results are significantly closer. The results also depend on the choice of $R$. Within the present truncation the variation with $R$ is of a similar order of magnitude [23].

\begin{tabular}{c||c|c|c||c|c||c}
$N$ & $A$ & $A^{\prime}$ & $A^{\prime \prime}$ & $B^{\prime}$ & $B^{\prime \prime}$ & $C^{\prime}$ \\
\hline-2 & $\frac{1}{2}$ & $\frac{1}{2}$ & $\frac{1}{2}$ & $\frac{1}{2}$ & $\frac{1}{2}$ & $\frac{1}{2}$ \\
0 & .592 & .583 & .649 & .565 & .574 & .582 \\
1 & .650 & .626 & .738 & .587 & .607 & .626 \\
2 & .708 & .666 & .825 & .605 & .636 & .669 \\
3 & .761 & .699 & .901 & .620 & .663 & .710 \\
4 & .804 & .725 & .962 & .631 & .688 & .749 \\
$\infty$ & 1 & .828 & 1.21 & 1 & 1 & 1
\end{tabular}

Table 1: Critical exponents $\nu$ to leading order in the derivative expansion from various flows.

On a technical level, the integration of the flows $A, B$ and $C$ is significantly more difficult than the standard flow $X=0$ or the primed flows, because they involve higher order flow terms on their right-hand sides. For the flow $A$, this dependence is simple enough to be diagonalised explicitly [23]. For the flow $B$, the study is simplified by noticing that the fixed point solution of $B$ and $B^{\prime \prime}$ are identical. Once the fixed point solution is found, the critical 
exponents can be computed directly. For the computations, we make use of a polynomial approximation, either about vanishing field or the minimum of the potential. The critical exponents showed good convergence except for the flow $B$, where no definite result has been found within the polynomial expansion.

Consider the cases $A, A^{\prime}$ and $A^{\prime \prime}$. By construction, all $A$-flows automatically fulfil the requirement (9). Comparing the results for $A$ and $A^{\prime}$, we conclude that the intrinsic scaling contributes between $2-10 \%$ to the value for $\nu$. Notice that the values in the second row are slightly closer to the physical values [27], despite the fact that the underlying approximation is less accurate than the one leading to the flow $A$. In the flow $A^{\prime \prime}$, we have neglected only the term in (19) proportional to $\partial_{t}\left(m_{k}^{2} / k^{2}\right)$. In comparison to $A$ and $A^{\prime}$, we notice a strong increase for the critical exponent about $10-20 \%$. Conversely, retaining only the term $\propto \partial_{t}\left(m_{k}^{2} / k^{2}\right)$ of (19) in the flow would have lead to a decrease of $\nu$ by roughly the same amount (data not displayed). Hence, we found that both terms of (19) contribute strongly and with opposite sign to universal quantities. We also notice that the limit $N=-2$ more stable than the large- $N$ limit, which is not reproduced by $A^{\prime}$ and $A^{\prime \prime}$. This shows that maintaining (9) and the correct large- $N$ limit is not compatible with the approximations (20) or (22).

Next we consider the flows $B^{\prime}$ and $B^{\prime \prime}$. By construction, all $B$-flows are subject to the approximation (9). The $B$-flows are sensitive to a non-trivial background field dependence of the regulator. The flows $B^{\prime}$ and $B^{\prime \prime}$ reproduce the correct limits for $N=-2$ and $\infty$. This shows that the approximation (9) is compatible with (20) or (22) for background field dependent regulators, even in the large- $N$ limit. Numerically, the results for $B^{\prime}$ and $B^{\prime \prime}$ are below the physical values. Also, the values for $B^{\prime}$ are smaller than those for $B^{\prime \prime}$. This is qualitatively the same as for the $A$-flows.

Structurally, the flow $C^{\prime}$ differs from $B^{\prime}$ only in the choice for the regulator, which is given by (田) with (13) in the former, and by (15) with (18) in the latter. The flow $C^{\prime}$ differs from $A^{\prime}$ in the additional background field dependence. This affects the numerical values for the critical exponents. This does not affect the limits $N=-2$ or $N=\infty$, in agreement with the reasoning given above for the $B$-flows. The $C^{\prime}$ results agree with those given in 24, 19. It is very intriguing that the values obtained for $A^{\prime}$ and $C^{\prime}$ are both very close to the physical values, and very close to each other, ranging within $.2-3 \%$ for $N \in[0,4]$. In the light of our discussion above, we expect that the additional terms found in (12) should modify the results for the critical indices.

\section{Conclusions}

We have studied the implications of background field dependent flows. Conceptually, the rôle of $\Gamma^{(2,0)}$ has been threefold. First of all, its appearance in the regulator has lead to an important simplification. The background field flow becomes essentially diagonal 
leading to a natural expansion in the eigenfunctions of $\Gamma^{(2,0)}$. Secondly, the deviation from this property is proportional to $\partial_{t} \Gamma^{(2,0)}$ leading to additional flow terms. Their numerical treatment was more difficult than the standard flow. Thirdly, the regulator cuts off both large momentum and large field amplitude contributions to the flow. We expect that the background field flows presented here are useful for examining 'local' properties such as critical exponents as done in the present case. Global flows have to be studied separately as more care is required concerning the irrelevance of the neglected terms.

Numerically, a coherent picture has emerged as well. The implicit dependences leave the qualitative result unaffected, supporting the viability of the approximation (9). Still, the corrections are quantitatively large and cannot be neglected for high precision computations. Best agreement with experimental data is achieved for the flows $A^{\prime}$ and $C^{\prime}$, despite the fact that implicit dependences are suppressed in both cases. This deserves further study, in particular in view of the full background field dependence.

More work has to be done for a study of non-universal quantities and for applications to non-Abelian gauge theories. There, most notably, the anomalous dimension of the gauge field is far from being small, the latter being a property coming to our help in the present work.

\section{Acknowledgements}

JMP thanks CERN for hospitality and financial support. The work of DFL has been supported by the European Community through the Marie-Curie fellowship HPMF-CT1999-00404.

\section{References}

[1] M. Reuter and C. Wetterich, Nucl. Phys. B417 (1994) 181.

[2] M. Reuter and C. Wetterich, Phys. Rev. D 56 (1997) 7893 hep-th/9708051.

[3] M. Reuter, Phys. Rev. D 53 (1996) 4430 hep-th/9511128].

[4] M. Reuter, Mod. Phys. Lett. A 12 (1997) 2777 hep-th/9604124].

[5] J. M. Pawlowski, Phys. Rev. D58 (1998) 045011 hep-th/9605037.

[6] D. F. Litim and J. M. Pawlowski, Phys. Lett. B435 (1998) 181 [hep-th/9802064]; Nucl. Phys. Proc. Suppl. 74 (1999) 329 hep-th/9809023]; Nucl. Phys. Proc. Suppl. 74 (1999) 325 hepth/9809020].

[7] S. Falkenberg and B. Geyer, Phys. Rev. D58 (1998) 085004 hep-th/9802113.

[8] D. F. Litim and J. M. Pawlowski, hep-th/9901063.

[9] F. Freire and C. Wetterich, Phys. Lett. B 380 (1996) 337 hep-th/9601081.

[10] F. Freire, D. F. Litim and J. M. Pawlowski, Phys. Lett. B 495 (2000) 256 hep-th/0009110; Int. J. Mod. Phys. A 16 (2001) 2035 hep-th/0101108. 
[11] J. M. Pawlowski, Int. J. Mod. Phys. A 16 (2001) 2105; in preparation.

[12] M. Bonini and E. Tricarico, Nucl. Phys. B 606 (2001) 231 hep-th/0104255.

[13] H. Gies, Phys. Rev. D 66 (2002) 025006 hep-th/0202207.

[14] D. F. Litim and J. M. Pawlowski, hep-th/0203005.

[15] M. Reuter and C. Wetterich, Nucl. Phys. B 427 (1994) 291.

[16] B. Bergerhoff, D. F. Litim, S. Lola and C. Wetterich, Int. J. Mod. Phys. A11 (1996) 4273;

B. Bergerhoff, F. Freire, D. F. Litim, S. Lola and C. Wetterich, Phys. Rev. B53 (1996) 5734;

F. Freire and D. F. Litim, Phys. Rev. D 64 (2001) 045014 hep-ph/0002153.

[17] M. Reuter, Phys. Rev. D 57 (1998) 971 hep-th/9605030;

O. Lauscher and M. Reuter, Phys. Rev. D 65 (2002) 025013 hep-th/0108040;

M. Reuter and F. Saueressig, Phys. Rev. D 65 (2002) 065016 hep-th/0110054.

[18] S. B. Liao, Phys. Rev. D56 (1997) 5008 hep-th/9511046; Phys. Rev. D53 (1996) 2020 hepth/9501124.

[19] D. F. Litim and J. M. Pawlowski, Phys. Lett. B 516 (2001) 197 hep-th/0107020].

[20] D. F. Litim and J. M. Pawlowski, Phys. Rev. D 65 (2002) 081701 hep-th/0111191.

[21] D. F. Litim and J. M. Pawlowski, Phys. Rev. D 66 (2002) 025030 hep-th/0202188.

[22] D. F. Litim, Phys. Lett. B486 (2000) 92 hep-th/0005245; Phys. Rev. D 64 (2001) 105007 hep-th/0103195; Int. J. Mod. Phys. A 16 (2001) 2081 hep-th/0104221; Phys. Lett. B393 (1997) 103 hep-th/9609040.

[23] D. F. Litim, Nucl. Phys. B 631 (2002) 128 hep-th/0203006.

[24] M. Mazza and D. Zappalà, Phys. Rev. D 64 (2001) 105013 hep-th/0106230.

[25] O. Bohr, B. J. Schäfer and J. Wambach, Int. J. Mod. Phys. A 16 (2001) 3823 hep-ph/0007098.

[26] A. Bonanno and D. Zappalà, Phys. Lett. B504 (2001) 181 hep-th/0010095.

[27] D. F. Litim, JHEP 0111 (2001) 059 hep-th/0111159. 